\title{
Near infrared imaging of the cometary globule CG $12^{\star, \star \star}$
}

\author{
L. K. Haikala ${ }^{1}$ and B. Reipurth ${ }^{2}$
}

\author{
1 Observatory, PO Box 14, University of Helsinki, Finland \\ e-mail: lauri.haikala@helsinki.fi \\ 2 Institute for Astronomy, University of Hawaii, 640 N. Aohoku Place, Hilo, HI 96720, USA \\ e-mail: reipurth@ifa.hawaii.edu
}

Received 7 August 2009 / Accepted 15 October 2009

\section{ABSTRACT}

\begin{abstract}
Context. Cometary globule 12 is a relatively little investigated medium- and low mass star forming region 210 pc above the Galactic plane.

Aims. This study sets out to discover the possibly embedded members of the CG 12 stellar cluster, to refine the NIR photometry of the known member stars and to study the star formation activity in CG 12 and its relation to the distribution of molecular gas, dust and mid- to far-infrared emission in the cloud.

Methods. NIR $J, H$, and $K$ s imaging and stellar photometry is used to analyse the stellar content and the structure of CG 12 .

Results. Several new members and member candidates of the CG 12 stellar cluster were found. The new members include in particular a highly embedded source with a circumstellar disk or shell and a variable star with a circumstellar disk which forms a binary with a previously known A spectral type cluster member. The central source of the known collimated molecular outflow in CG 12 and an associated "hourglass"-shaped object due to reflected light from the source were also detected. The maximum visual extinction in the cloud, based on observations of background stars, is $\sim 20^{\mathrm{m}}$, but this is only a lower limit for the extinction through the two dense cloud cores. HIRES-enhanced IRAS images are used together with $J H K$ s imaging to study the two associated IRAS point sources, 13546-3941 and 13547-3944. Two new $12 \mu \mathrm{m}$ sources coinciding with NIR excess stars were detected in the direction of IRAS 13546-3941. The IRAS 13547-3944 emission at 12 and $25 \mu$ m originates in the Herbig AeBe star h4636n and the 60 and $100 \mu \mathrm{m}$ emission from an adjacent cold source.
\end{abstract}

Key words. stars: formation - stars: pre-main-sequence - ISM: individual objects: CG 12 - ISM: individual objects: NGC 5367 infrared: stars

\section{Introduction}

Low and intermediate mass star formation takes place primarily in isolated clusters (e.g., the CrA star forming cloud) and to a lesser extent in low mass star forming regions (e.g., Taurus and Chamaeleon). As the stars form in dense gas/dust clouds, which are tightly concentrated towards the Galactic plane, star formation far out of the plane is not a likely event. Even though Chamaeleon and $\mathrm{CrA}$ are high Galactic latitude regions, they lie well within the scale height of molecular interstellar matter in the Galaxy (50-75 pc). The well known nearby star forming regions have been studied in great detail from visual to radio wavelengths, but less is known of star formation regions off the Galactic plane.

van Till et al. (1975) detected strong CO emission in the direction of the reflection nebulosity NGC 5367 and, assuming a distance of $300 \mathrm{pc}$, calculated a mass of $30 M_{\odot}$ for the mapped area. NGC 5367 lies in the head of the Cometary Globule 12, CG 12, listed in Hawarden \& Brand (1976). Despite being classified by Hawarden \& Brand (1976) as a cometary globule, together with those in the Gum nebula (Reipurth 1983), CG 12

* Based on observations collected at the European Southern Observatory, La Silla, Chile.

$\star \star$ Table 1 is only available in electronic form at the CDS via anonymous ftp to

cdsarc.u-strasbg.fr $(130.79 .128 .5)$ or via

http://cdsweb.u-strasbg.fr/cgi-bin/qcat?]/A+A/510/A1 is actually a high-latitude low- and intermediate-mass star formation region. With a Galactic latitude of $21^{\circ}$ and at the distance of $\sim 550 \mathrm{pc} \quad$ (Maheswar et al. (1996) and Getman et al. (2008), hereafter GFLBG), CG 12 lies more than 200 pc above the plane. A loose stellar cluster including at least three late B stars and two A stars (Williams et al. 1977) is associated with CG 12. The associated reflection nebula NGC 5367 is illuminated by the binary star h4636 (spectral types B4V and B7V). This binary contains at least one, or possibly two, Herbig AeBe stars (Reipurth \& Zinnecker 1993). GFLBG find a high concentration of X-ray sources in the CG1̃2 region mainly in the direction of visible or NIR stellar objects and conclude that more than 50 of these are T-Tauri stars associated with the nebula. White (1993) mapped the CG 12 centre region in CO (2-1) and $\mathrm{C}^{18} \mathrm{O}(2-1)$ and found a compact core and a highly collimated molecular outflow. Further molecular line observations of CG 12 are presented in Yonekura et al. (1999) and recently in Haikala \& Olberg (2007) (Paper 1) and Haikala et al. (2006) (Paper 2). The structure and dynamics of the CG 12 core have been discussed in detail in Papers 1 and 2. The molecular material lies in a $10^{\prime} \mathrm{NS}$ elongated lane with three compact $\mathrm{C}^{18} \mathrm{O}$ maxima, CG 12-N, CG 12-S, and CG 12-SW. The southern maximum, CG 12-S, lies near the binary $\mathrm{h} 4636$. The $\mathrm{C}^{18} \mathrm{O}$ emission in CG 12-S traces mainly warm gas on the surface of a dense core (called $\mathrm{DCO}^{+}$core) revealed in $\mathrm{DCO}^{+}$and $\mathrm{CS}$ emission and in $1.2 \mathrm{~mm}$ continuum (Paper 1; Paper 2). The centre of the highly collimated molecular outflow reported in White (1993) lies in 
the direction of the $\mathrm{DCO}^{+}$core. The northern core, $\mathrm{CG} 12-\mathrm{N}$, is cold and the relative velocities and intensities of $\mathrm{C}^{18} \mathrm{O}$ and high density tracers indicate that molecular material is highly depleted. The molecular mass of the CG 12 core area, as traced by the $\mathrm{C}^{18} \mathrm{O}$ observations, is larger than $100 M_{\odot}$. This mass corresponds only to the area observed in $\mathrm{C}^{18} \mathrm{O}$ and traced by the $\mathrm{C}^{18} \mathrm{O}$ emission. The mass of the cloud envelope not visible in $\mathrm{C}^{18} \mathrm{O}$ and that outside the mapped area is much larger, as shown by e.g. the large scale CO mapping of Yonekura et al. (1999) according to which the mass of the globule is larger than $300 M_{\odot}$. The linear size of the CG 12 core region (>1 pc) and the molecular mass are of the same order as those of known nearby low mass star formation regions like Chamaeleon I. CG 12 has been recently reviewed by Reipurth et al. (2008).

The median photometric age of the T-Tauri population in CG 12 is $\sim 4$ Myr, but the apparent spread of ages is considerable, from 1 to $20 \mathrm{Myr}$ (GFLBG). The more than 50 T-Tauri stars detected by GFLBG represent possibly the first generation of stars born in the CG 12 area, and the B and A stars (Williams et al. 1977) may be the second generation. The four IRAS point sources and the collimated molecular outflow in the CG 12 cloud show that star formation is presently going on. Because of the great distance and the high extinction towards the cloud core, the limiting magnitudes of the Two Micron All Sky Survey (2MASS) allow only the detection of the brightest stars embedded in the cloud in all three $J H K$ s colours. A deeper $J, H, K$ s survey of CG 12 than 2MASS is clearly needed to study the possible embedded stellar population. Spitzer NIR IRAC imaging data on CG 12 has recently become public and can also be used to analyse the embedded stars.

We report imaging of the core of $\mathrm{CG} 12$ in $J, H$, and $K$ s NIR bands with SOFI at the NTT telescope at La Silla. Observations, data reduction, and calibration procedures are described in Sect. 2 and the observational results in Sect. 3. The new results are discussed and compared with available data at other wavelengths in Sect. 4. The results are summarised and the conclusions drawn in Sect. 5.

\section{Observations and reductions}

CG 12 was imaged in $J, H$, and $K$ s with SOFI on the NTT during two observing runs, May 20-24, 2005 and June 13-15, 2007. In 2007 the $J$ s filter was used instead of the $J$. In order to optimise the coverage in the north-south direction the detector array was rotated 45 degrees. Because of the high surface brightness in CG 12 the observations were carried out in the on-off mode instead of the standard jitter mode. After each on-integration an off-position outside the globule was observed and jittering was done after each on-off pair. The $2005 \mathrm{H}$ and $\mathrm{K}$ s integrations consisted of 10 individual $6 \mathrm{~s}$ integrations. The $2005 \mathrm{~J}$ and all the 2007 observations had a $10 \mathrm{~s}$ basic integration time. The average

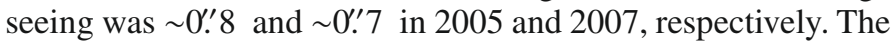
observed on-source times were 40, 24, and $30 \mathrm{~min}$ in 2005 and 65,24 , and $41 \mathrm{~min}$ in 2007 in the $J, H$, and $K$ s, respectively. Standard stars from the Persson et al. (1988) faint NIR standard list were observed frequently during the nights.

The IRAF $^{1}$ external XDIMSUM package was used in the data reduction. The images were searched for cosmic rays, sky subtracted, flat fielded, illumination corrected, registered and averaged. The two off-position images nearest in time to each

1 IRAF is distributed by the National Optical Astronomy Observatories, which are operated by the Association of Universities for Research in Astronomy, Inc., under cooperative agreement with the National Science Foundations. on-position image were used in the sky-subtraction. An object mask was constructed for each off-position image. Applying these masks in the sky-subtraction produced hole-masks for each sky-subtracted image. Special dome flats and illumination correction frames provided by the NTT team were used to flat-field and to illumination-correct the sky-subtracted images. Rejection masks combined from a bad pixel mask and individual cosmic ray and hole masks were used when averaging the registered images.

A bright star in an image produces an artifact (inter-quadrant row cross talk) which appears as a stripe at the same line as the star and symmetrically on the other half of the detector. This can be corrected if the star in question is not saturated (see the SOFI manual). However, in CG 12 both the components of the binary h4636 are heavily saturated and the striping cannot be corrected. Reflections in the $H$ filter cause ring-like ghosts around bright stars. No effort was made to correct this.

The intensity scale was monitored using the standard star observations before and after each observing block. The 2007 $J$ s counts were $5 \%$ smaller than the $2005 J$ counts. The $2007 J \mathrm{~s}$ data were scaled to bring the data sets into the same scale. The possible slight colour-dependent difference due to the narrower $J$ s filter was ignored.

The SExtractor software v. 2.5.0 (Bertin \& Arnouts 1996) package was used to detect the stars and galaxies in the images. Altogether 423 sources were detected in all the three filters. The galaxies were excluded both by using the SExtractor star keyword and by visual inspection of the images. Strong surface brightness filaments also appeared as extended and elongated sources in the photometry. After elimination of sources estimated to be galaxies and sources due to surface brightness, 280 sources remained. Of these 218 have formal errors less than 0.15 in all colours. If only $H$ and $K$ s filters are considered the number amounts to 227. The magnitude zero points of the added-up data were fixed using the standard star measurements from the 2005 observing run. The instrumental magnitudes were first converted to the Persson et al. (1988) photometric system and then to the 2MASS photometric system as described in Ascenso et al. (2007). Finally, the magnitude scale was checked by comparing the SOFI photometry of stars in common with 2MASS and which had good quality 2MASS photometry. Apart from the photometry zero point check the transformation to the 2MASS photometric system was done to make the comparison with literature data easier. The limiting magnitudes (for a formal error of 0.15 ) are approximately $21^{\mathrm{m}} 0,20^{\mathrm{m}} 5$ and $20^{\mathrm{m}} 0$ for $J, H$, and $K \mathrm{~s}$, respectively. The limiting magnitude, however, varies over the observed area and is less in the regions of strong surface brightness and also where the brightness gradient is strong. This concerns especially the surroundings of the binary h4636.

The components of the binary h4636 were strongly saturated and no photometry was possible. The counts of some of the brightest stars in the field were within the saturation limit. The $H$ and $K$ s photometry for these stars was obtained using the 2005 observations with a $6 \mathrm{~s}$ basic integration time. Even if the stars were not saturated their count levels were in the nonlinear region of the detector. An extreme nonlinearity of 5\% would produce an error of 0.05 . This will be noted below where the photometry is discussed. The photometry of the two close binaries, the Williams et al. (1977) star W77-6 and the one in the direction of CG $12-\mathrm{N}$, is challenging because of the brightness of the stars and the strong background nebulosity. These stars were measured manually by varying both the source and background apertures to reach a satisfactory compromise. As a check, the chosen aperture settings were also applied to single bright 


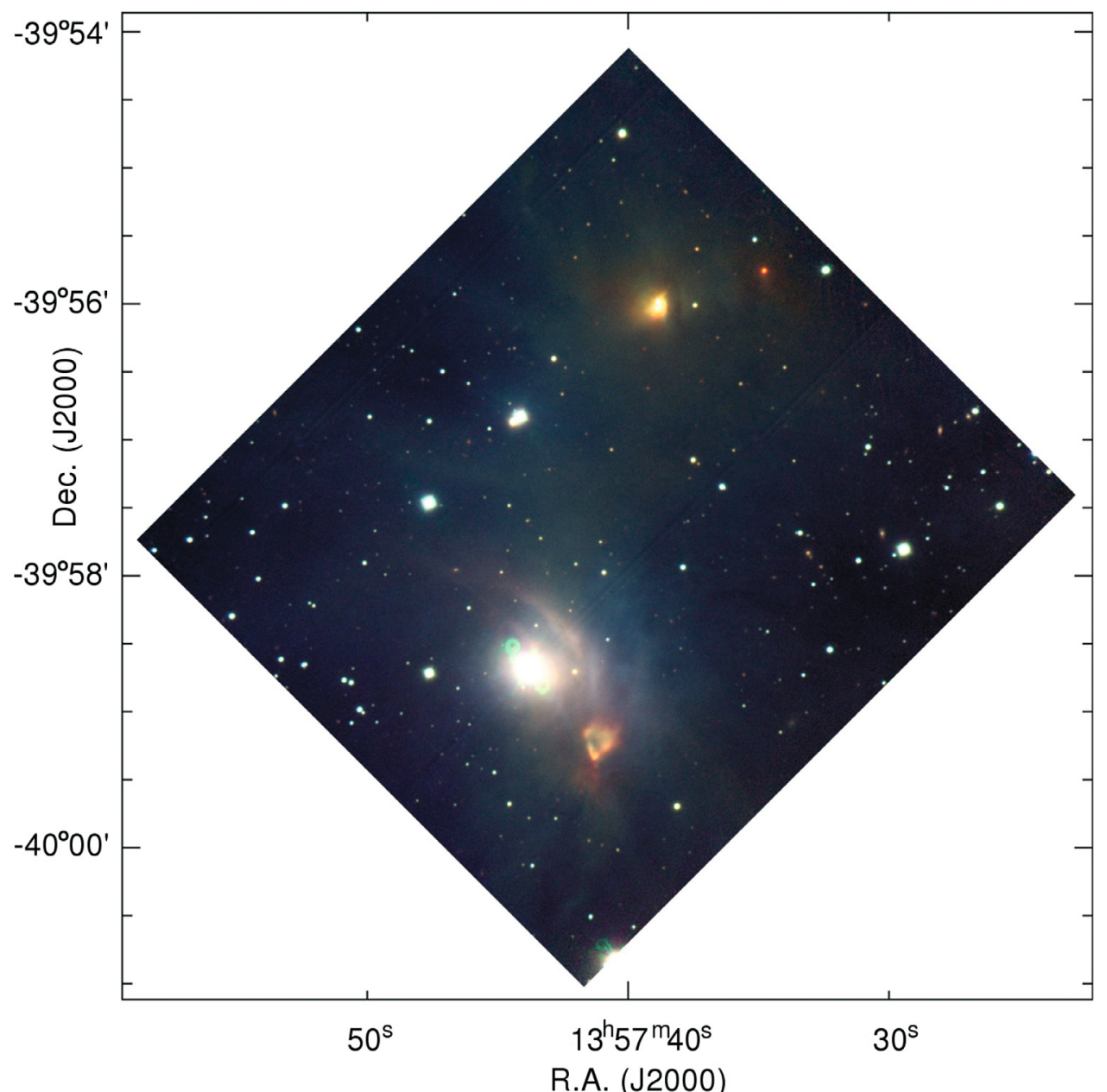

Fig. 1. Colour coded SOFI image of CG 12 . The $J, H$, and $K$ s bands are coded in blue, green and red, respectively. Square root scaling has been used to better bring out the faint surface brightness structures.

stars, the results of which were compared with those from the automated SExtractor photometry. Even though the single stars agreed well, one should bear in mind that the uncertainty in the binary photometry exceeds the formal error of the measurement.

The results of the SOFI stellar photometry are tabulated ordered by increasing right ascension in the online Table 1. Columns one to three list our stellar sequence number, other ID, and the $\mathrm{J}(2000)$ coordinates, respectively. The $J, H$, and $K$ s magnitudes with their formal errors and the $(J-H),(H-K s)$ colours are listed in columns four to eight, respectively. Comments are given in column nine. The stars considered as members or potential members of the CG 12 stellar cluster (see Sects. 4.1.1 to 4.1.7) are identified in the comments column with 1 and 2, respectively. The GFLBG Chandra X-ray source designation I_NN, where I stands for the GFLBG field I and NN the sequence number, is also given for stars that are the same as in that publication. For the identification with the Chandra objects a positional coincidence better than $1^{\prime \prime}$ was required for stars not already identified with 2MASS stars by GFLBG.

\section{Results}

\subsection{Imaging}

The on-off observing method used in this study preserves also the extended surface brightness which is smeared out if the standard jitter observing method is used. Only the surface brightness gradients are visible in the final reduced images if the simple jitter method is used. Extended surface brightness due to scattered light is seen in all three colours. However, it is not possible to determine the absolute level of this surface brightness. Therefore, the surface brightness level was arbitrarily set to zero in a small region in the western part of the images where visual inspection shows that the surface brightness is the smallest. However, the real background zero level, even in the $K$ s band where it is the lowest, most probably lies outside the imaged area.

A false colour SOFI image of CG 12 is shown in Fig. 1. The $J, H$, and $K$ s images are coded in blue, green, and red respectively. The image is dominated by the bright binary $\mathrm{h} 4636$ and the reflection nebulosity it is illuminating. The reflection nebulosity surrounding h4636 is predominantly bluish in colour whereas the northern part of the image is reddish. The number of stars (and galaxies) in the western and eastern parts of the image is high compared to the north-south diagonal where at some locations hardly any stars are seen. The number density of stars anticorrelates, as expected, with the observed $\mathrm{C}^{18} \mathrm{O}$ emission in the globule (Paper 1). Other noteworthy features are the cone shaped object to the SW of h4636, a nebulous binary in the northern part of the globule, and a dark lane to the West of it. A very red object (star 58) lies west of this binary near the edge of the image. An elongated cavity, best visible in the $J$ image, is seen north of the cone. The location of these features in the SOFI image and selected stellar objects to be discussed below are shown in Fig. 2. The positions of these objects relative to the 


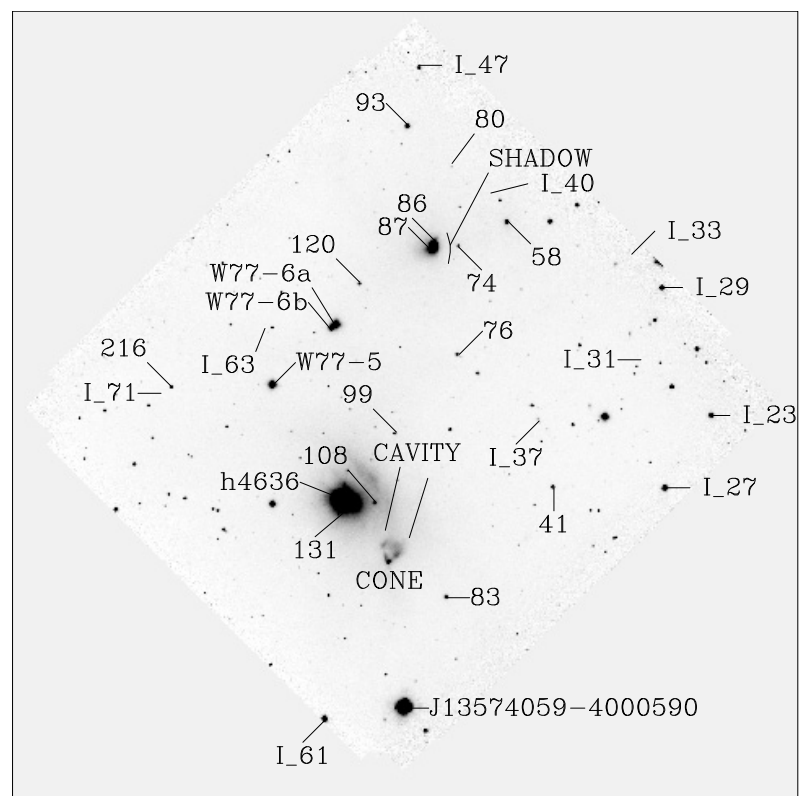

Fig. 2. SOFI $K$ s band image. Notable features in the cloud are identified. The stars in the online Table 1 are identified with their sequence number. W77-5 and -6 refer to the Williams et al. (1977) identification. The 2MASS J13574059-4000590 and the GFLBG objects (I_NN) not in Table 1 are also indicated.

IRAS point sources, the $\mathrm{C}^{18} \mathrm{O}$ cores (CG $12-\mathrm{S}$ and $\mathrm{CG} 12-\mathrm{N}$ ), the $\mathrm{DCO}^{+}$emission and the ${ }^{12} \mathrm{CO}$ molecular outflow (White 1993) are shown in Fig. 10 in Paper 1. The cone is projected on the $\mathrm{DCO}^{+}$core and the centre of the collimated molecular outflow (White 1993) but it lies south of the $\mathrm{C}^{18} \mathrm{O}$ maximum. The nebulous binary in the North lies at the edge of CG 12-N.

\subsection{Photometry}

The $J-H / H-K$ s colour-colour diagram for stars with $J, H$, and $K$ s formal errors less than 0.15 is shown in Fig. 3. The only star in the image with a larger error in $J$ is the highly reddened star 58. The main-sequence, the giant branch (Bessell \& Brett 1988) and the M0 CTTS-locus (Meyer et al. 1997), all transformed to the 2MASS photometric system, are plotted in red, yellow, and blue respectively. The dashed lines show the effect of visual extinction in the diagram according to the Bessell \& Brett (1988) reddening law, with the red arrow corresponding to $A_{\mathrm{v}}$ of $10^{\mathrm{m}}$. It is assumed in the figure that the slope of the reddening line is constant even for high extinctions. A small symbol is used if the $(J-H)$ and/or the $(H-K \mathrm{~s})$ formal error is larger than 0.1 . The stars with $K$ s magnitudes 15 or less are indicated with red circles. The blue diamonds indicate X-ray detections (GFLBG) associated with stellar objects in the SOFI image. The $J-H / H-K$ s diagram includes the Williams et al. (1977) stars 2 and 8 and the star 2MASS J13574059-4000590, which are not inside Fig. 1. The colours for these stars, the Williams et al. (1977) star 5 and for h4636s, which were saturated in the SOFI images, were taken from the 2MASS catalog. The $(H-K \mathrm{~s})$ index of stars which had reliable photometry only in the $H$ and $K \mathrm{~s}$ bands are indicated with green error bars at the bottom of Fig. 3 .

Even though the obvious extragalactic sources were filtered out from the original data set, it is highly likely that some of these sources still remain in the final data set. Likely candidates are e.g. the faint outliers below the CTTS locus and to the right of the reddening line and objects with negative $(H-K \mathrm{~s})$ colour.

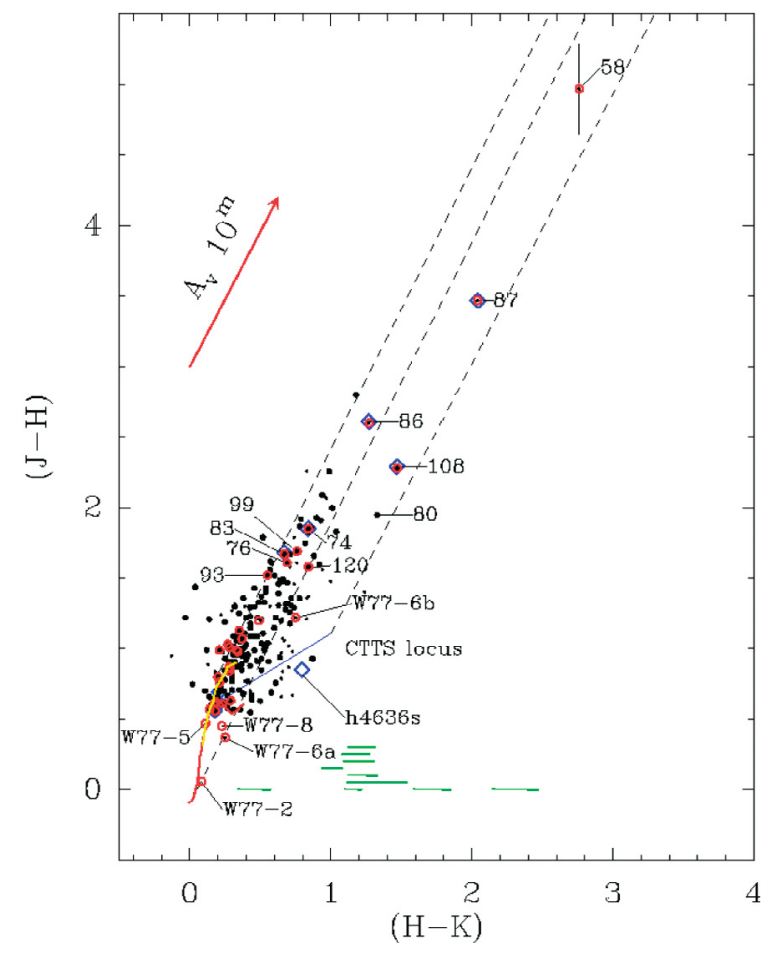

Fig. 3. $J-H / H-K$ s diagram for stars with formal $J H K$ s errors less than 0.15 . The stars with $(J-H)$ and/or $(H-K \mathrm{~s})$ error larger than $0^{\mathrm{m}} 1$ are plotted using a small symbol. The stars for which the $J$ error is large but which are well detected in $H$ and $K$ s are indicated with horizontal green $(H-K \mathrm{~s})$ error bars at the bottom of the plot. The main-sequence, the giant branch and the M0 CTTS-locus are plotted in red, yellow and blue respectively. Blue diamonds indicate stars detected in X-ray (GFLBG). Red circles identify the stars which are brighter than $15^{\mathrm{m}} .0$ in $K \mathrm{~s}$. The dashed lines show the effect of visual extinction in the diagram according to the Bessell \& Brett (1988) reddening law, with the red arrow corresponding to $A_{\mathrm{v}}$ of $10^{\mathrm{m}}$.

\section{Discussion}

The new NIR imaging and photometry of the CG 12 head, together with data available at other wavelengths, is used to analyse the associated embedded stellar population. The data additional to the present NIR observations are in particular from the 2MASS survey, the data from the IRAS satellite, the Chandra X-ray data (GFLBG), and Spitzer NIR imaging. In the following the NIR photometry is discussed first, then the extended surface emission and finally, the IRAS observations.

\subsection{Photometry}

The distance to CG 12 is uncertain. Maheswar et al. (1996) and GFLBG, using different methods, suggest a distance of $550 \mathrm{pc}$. This is less than the $630 \mathrm{pc}$ obtained by Williams et al. (1977) using main sequence fitting. The distance modulus ranges therefore between 8.6 and 9.0 . The strong extinction in the direction of CG 12 starts at the distance of CG 12 (Maheswar et al. 1996). One would therefore expect to see not only stars embedded in the cloud as well as background stars but also unreddened foreground objects. The number of these foreground stars can be estimated as follows. Mattila (1980) gives the number of main sequence stars from late $\mathrm{O}$ to $\mathrm{M}$ spectral types and giants from $\mathrm{F} 8$ to M8 as a function of spectral type and Galactic latitude in the local Milky Way. The estimated number of CG 12 foreground stars in a $5^{\prime}$ by $5^{\prime}$ field, at a Galactic latitude of $21^{\circ}$ is $\sim 6$. The 
expected foreground stars are of spectral type G5 or later and none earlier. The number of giant stars in the field (including also the background) is predicted to be smaller than 1. As argued by GFLBG, the number of the known B and A type stars associated with $C G 12$ predicts a population of $\sim 80$ stars with masses $0.5 M_{\odot}<M<2 M_{\odot}$. The SOFI image should therefore contain a considerable fraction of this unknown population, and if not too deeply embedded, they should be visible in the image. The Mattila (1980) model does not predict any early type field stars other than by chance at this high Galactic latitude region. The early type stars seen in CG 12 are therefore likely to be members of the CG 12 stellar population.

The unreddened foreground stars are likely to be among the brightest stars in the SOFI field. The absolute $K$ magnitude of late main sequence $M$ stars is less or equal to $5^{\mathrm{m}}$ (Bessell \& Brett 1988). An unreddened G0 or M5 main-sequence star at a distance of $550 \mathrm{pc}$ has a $K$ magnitude of $11^{\mathrm{m}} 6(12 \mathrm{~m} 0$ in $J)$ and $14.7(15.7$ in $J)$ respectively. This indicates that stars fainter than 15 . 0 in $K$ are either embedded in CG 12, or are background objects. Stars brighter than this can be either foreground objects, embedded in CG 12 or background stars. The stars brighter than $15^{\mathrm{m}}$ in $K$ in the SOFI field are indicated by red circles in Fig. 3. As predicted by the Galactic stellar model some of these stars have non-reddened late main-sequence colours. A larger group of these stars have colours indicating extinction less than $5^{\mathrm{m}}$ in the visual (assuming they are not early-type main-sequence stars). Many of these could be background F-G type stars. A group of bright stars is located around $(J-H) 1.7,(H-K s) 0.8$ in Fig. 3 indicating a visual extinction of $10^{\mathrm{m}}$ or larger. Stars 74 and 120 are among these stars. It is not possible from the $J H K$ s photometry alone to decide if these stars are embedded or background stars. Ten stars in the direction of the CG 12 cloud have reliable photometry only in $H$ and $K \mathrm{~s}$ bands (error $<0$. 15 in both bands). The $(H-K \mathrm{~s})$ colours of these stars are indicated by the green $(H-K \mathrm{~s})$ error bars in the lower part of Fig. 3. With the exception of one object the visual extinction towards these stars is likely to be $15^{\mathrm{m}}$ or more.

\subsubsection{Star 58 (J 135734.76-395543.8)}

This is the reddest object in the cloud. The star is only marginally detected in $J\left(21^{\mathrm{m}} .62 \pm 0.32\right)$ but is bright in $H$ and $K \mathrm{~s}\left(16^{\mathrm{m}} .61\right.$ and 13 . 89 , respectively). Its position in the $J-H / H-K$ s diagram indicates IR excess and reddening corresponding to an $A_{\mathrm{v}}$ of $35^{\mathrm{m}}$ or more. Because of the large error in the $(J-H)$ index the star could also be a highly reddened giant. If the star were a background giant it would, however, be difficult to explain the origin of the reddening. The $\mathrm{C}^{18} \mathrm{O}(1-0)$ integrated line intensity in the direction of this star is $2.5 \mathrm{~K} \mathrm{~km} \mathrm{~s}^{-1}$ in the main beam temperature scale (it is significantly offset from the $\mathrm{C}^{18} \mathrm{O}$ column density maximum, CG 12-N (Paper 1)) corresponding to $20^{\mathrm{m}}$ of $A_{\mathrm{v}}$. This is only half of the extinction deduced from the $\mathrm{J}-\mathrm{H} / \mathrm{H}-\mathrm{K}$ s diagram. There is also no hint of a dense core where $\mathrm{CO}$ could be depleted in the SIMBA $1.2 \mathrm{~mm}$ continuum map (Fig. 3, Paper 2). By chance the position in CG 12 where Bourke et al. (1995) observed $\mathrm{NH}_{3}$ is only $20^{\prime \prime}$ north of the position of this star and therefore well within the 1.'4 beam. The observed $0.11 \mathrm{~K} \mathrm{NH}_{3}$ antenna temperature does not indicate the presence of a localised dense core at this position.

One should, however, be very careful when assuming a one to one correspondence of the calculated $\mathrm{C}^{18} \mathrm{O}$ column density and optical extinction because the relation is statistical. The calculated $\mathrm{C}^{18} \mathrm{O}$ column density depends strongly on the assumed LTE condition, the adopted excitation temperature, the assumed beam filling factor and the molecular abundance used in the calculations (Paper 1). The $\mathrm{C}^{18} \mathrm{O}$ maximum $\mathrm{CG}$ 12-S highlights this problem. The $\mathrm{C}^{18} \mathrm{O}$ emission does not trace the maximum column density, which lies in the direction of the $\mathrm{DCO}^{+}$core, but warm gas on its surface (Paper 1; Paper 2). The molecular line observations of optically thin transitions like $\mathrm{C}^{18} \mathrm{O}$ trace the total cloud column density. There is, however, no justification for the assumption that a star embedded in the cloud and seen in the direction of a molecular column density maximum necessarily lies behind the maximum. The extinction towards a star corresponds to a pencil beam whereas the $\mathrm{C}^{18} \mathrm{O}$ column densities (and column densities of other molecules) are calculated from observations made with a gaussian beam and represent therefore an average over the beam. The half power beam widths of the CG $12 \mathrm{C}^{18} \mathrm{O}$ observations presented in Paper 1 are $47^{\prime \prime}$ and $24^{\prime \prime}$ for the (1-0) and (2-1) transitions respectively. At the assumed distance of CG $12,550 \mathrm{pc}, 47^{\prime \prime}$ corresponds to $0.125 \mathrm{pc}$.

The reddish surface brightness surrounding star 58 in Figs. 1 and 5 indicates that the star is embedded in the CG 12 cloud. Therefore, a plausible explanation for the high reddening is that the star is embedded in the CG 12 cloud and the reddening takes place in a circumstellar disk. Such a disk does not show up in single dish molecular line observations because of severe beam dilution. Star 58 is not detected in the Chandra observations (GFLBG) but is visible in the Spitzer IRAC images in all four bands from $3.6 \mu \mathrm{m}$ to $8 \mu \mathrm{m}$. The star is a Class I candidate embedded in the CG 12 cloud.

\subsubsection{Stars $74,86,87$ and 120}

Stars 74 (J 135737.49-395559.5), 86 (J 135738.92-395558.7), and 87 ( $\mathrm{J}$ 135738.95-395600.7) were detected in X-rays (GFLBG objects I_41, I_45, and I_46, respectively). Photometry for these stars is summarised in Table 2 where the SOFI/2MASS $J H K$ s photometry for stars discussed in detail in the text is listed. The position of star 74 in the $J-H / H-K$ s diagram shows that the visual extinction towards this star is $\sim 10 \mathrm{mag}$. It has no surrounding nebulosity, but its X-ray activity (GFLBG) indicates that it is likely to be associated with CG 12. In the Spitzer images it is visible only at the two shortest wavelengths.

Stars 86 and 87 form a nebulous binary in the direction of the $\mathrm{C}^{18} \mathrm{O}$ maximum $\mathrm{CG} 12-\mathrm{N}$. The approximate visual extinction towards stars 86 and 87 is larger than 15 and 20 mag respectively. This is significantly more than the $8-10$ mag estimated by GFLBG from their X-ray spectrum. Star 87 has NIR excess in Fig. 3. As discussed in Sect. 2 the photometry of these stars is difficult because of the surrounding background nebulosity and the brightness of star 87 in the $K$ s band. A further uncertainty in assessing the nature of star 87 is the uncertainty of the slope of the reddening curve and its colour dependence. However, the high extinction towards the two stars and the NIR excess of star 87 are not in doubt. In the Spitzer images the stars 86 and 87 are surrounded by a nebulosity. Both stars are saturated in all but the $8 \mu \mathrm{m}$ image.

The location of star $120(\mathrm{~J} 135743.10-395624.0)$ in the $J-H / H-K$ s diagram indicates a possible small infrared excess and approximately a visual extinction $A_{\mathrm{v}}$ of $7^{\mathrm{m}}$ if originating from the CTTS locus. The star is visible in all the Spitzer NIR images. There is no indication of nebulosity around the star in the $J H K$ s nor in the Spitzer images and it was not detected by Chandra (GFLBG). The de-reddened magnitudes suggest that the star is associated with CG 12. 
Table 2. JHKs photometry for selected stars in CG 12.

\begin{tabular}{lcccccc}
\hline \hline Star & $\begin{array}{c}J \\
\mathrm{mag}\end{array}$ & $\begin{array}{c}H \\
\mathrm{mag}\end{array}$ & $\begin{array}{c}K \mathrm{~s} \\
\mathrm{mag}\end{array}$ & $\begin{array}{c}(J-H) \\
\mathrm{mag}\end{array}$ & $\begin{array}{c}(H-K \mathrm{~s}) \\
\mathrm{mag}\end{array}$ & Comment \\
\hline 58 & $21.62 \pm 0.32$ & $16.65 \pm 0.01$ & $13.89 \pm 0.01$ & 4.97 & 2.76 & \\
74 & $17.62 \pm 0.01$ & $15.77 \pm 0.01$ & $14.93 \pm 0.01$ & 1.85 & 0.84 & \\
86 & $15.39 \pm 0.01$ & $12.67 \pm 0.01$ & $11.39 \pm 0.01$ & 2.72 & 1.28 & \\
87 & $16.63 \pm 0.01$ & $12.95 \pm 0.01$ & $10.81 \pm 0.01$ & 3.68 & 2.14 & \\
108 & $18.13 \pm 0.09$ & $15.85 \pm 0.05$ & $14.38 \pm 0.01$ & 2.29 & 1.47 & \\
120 & $16.59 \pm 0.02$ & $15.02 \pm 0.01$ & $14.18 \pm 0.01$ & 1.57 & 0.85 & \\
131 & $20.27:$ & $17.98 \pm 0.06$ & $16.69 \pm 0.03$ & 2.30 & 1.29 & \\
h4636s & $8.87 \pm 0.01$ & $8.07 \pm 0.09$ & $7.26 \pm 0.06$ & 0.80 & 0.81 & 2MASS \\
W77-5 & $11.19 \pm 0.01$ & $10.73 \pm 0.01$ & $10.69 \pm 0.01$ & 0.46 & 0.04 & 2MASS \\
W77-6a & $11.37 \pm 0.10$ & $10.95 \pm 0.10$ & $10.72 \pm 0.10$ & 0.42 & 0.23 & \\
W77-6b & $13.93 \pm 0.10$ & $12.79 \pm 0.10$ & $12.03 \pm 0.10$ & 1.14 & 0.76 & \\
\hline
\end{tabular}

\subsection{3. h4636, stars 108 and 131}

The components of the binary h4636 are the brightest objects in the SOFI image. Spectroscopy by Williams et al. (1977) showed that the northern component is a strong $\mathrm{H} \alpha$ emission line star while the southern component shows a pure absorption spectrum, and they suggested spectral types of B4V and $\mathrm{B} 7 \mathrm{~V}$, respectively. In the near infrared the northern component has excess emission due to circumstellar dust (Williams et al. 1977). Optical and/or NIR photometry is given by Williams et al. (1977), Marraco \& Forte (1978), Chelli et al. (1995) and 2MASS. The JHKLM photometry in Williams et al. (1977) includes both components. $J H K$ s photometry in 2MASS is available only for h4636s and only in $J$ for h4636n (the stars are bright and separated only by 3.'7). Chelli et al. (1995) were able to provide the $J H K$ s magnitude differences between the binary components from one dimensional specklegraph scan measurement. However, the magnitudes and magnitude differences from different dates do not agree. A plausible explanation for this is that one or both of the binary components are variable. Optical variability is suspected also by Williams et al. (1977). The 2MASS JHKs magnitudes for h4636s were taken simultaneously and its colour indices can be calculated. Its position in Fig. 3 indicates strong NIR excess. Both the components of h4636 are strong X-ray detections (I_56 and I_58, GFLBG).

Star 108 (J 135742.28-395846.7), which coincides with the X-ray source I_54 (GFLBG), lies west of h4636. The star has $J H K$ s colours indicative of NIR excess and a reddening corresponding to an $A_{\mathrm{v}}$ of more than $10 \mathrm{mag}$ if originating from the CTTS locus. GFLBG estimate an $A_{v}$ of $25 \mathrm{mag}$. It was the only source with significant variability during the Chandra observations (GFLBG). The star is visible in all but the $8 \mu \mathrm{m}$ Spitzer images. At $8 \mu \mathrm{m}$ the nebulosity at the position of the star becomes strong and this may be the reason why the star is not detected. Star 108 is a Class I candidate.

A faint star, 131 ( $\mathrm{J}$ 135744.32-395853.5), is detected south of $\mathrm{h} 4636$. The star is within the strong gradient of the bright h4636 nebulosity and the $J$ magnitude of the source is only an estimate. The star is detected in an $L$ band but not in an $M$ band ISAAC image (Yun 2008, private communication). It is not seen in the Spitzer images. The star is a potential member of the CG 12 stellar cluster.

\subsubsection{Williams et al. (1977) stars 2, 5, 6, and 8}

Williams et al. (1977) stars W77-2 (2MASS J135756014004192, CD-39 ${ }^{\circ} 8583$ ) and W77-8 (2MASS J135726093956091 ) are not within the NTT SOFI image but their $J, H$, and $K$ s magnitudes are available in 2MASS. Star W77-2 is a late B star (Maheswar et al. 1996) and W77-8 an A0 type star (Gahm \& Malmort 1980). Both stars are surrounded by optical nebulosities and are members of the CG 12 stellar cluster. The SOFI photometry of star W77-5 (2MASS J13574803-3957295) is in agreement with the 2 MASS values even though it lies near the saturation limit. 2MASS photometry is, however, adopted when calculating the colour indices. The star is positioned on the late main sequence in Fig. 3. This is in line with its spectral type K0 given in Gahm \& Malmort (1980) and its location in the $U B V$ colour-colour diagram (Williams et al. 1977). The star is a foreground object.

Star W77-6 is a binary (132, J 135744.43-395650.5 and 138, J 135744.70-395652.8) with a separation of $\sim 4^{\prime \prime}$. The only good quality 2MASS magnitudes are for the brighter binary component in $H$ and $K \mathrm{~s}$. The SOFI $(J-H)$ and $(H-K \mathrm{~s})$ colour indices are 0.37 and 0.25 for the brighter, northern (a) component (132, W77-6a) and 1. 20 and 0.75 magnitudes for the southern (b) component (138, W77-6b). Because of the brightness of star 132 in all three bands and 138 in the $K$ band they fall on the nonlinear part of the detector so the magnitudes in these bands and the colour indices derived are somewhat uncertain. Star W77-6b is strongly variable, and the photometry above is for the 2005 observations when single night $J H K$ s photometry is available. The $J$ magnitude of star W77-6b varied from 13.90 to 14.20 between 2005 and the first observation in 2007. In a single night in 2007 the $J$ magnitude changed from 14.20 to 14.84 . This is far more than can be explained by the uncertainty introduced by the background nebulosity and detector nonlinearity. The $H$ and $K$ s magnitudes in 2005 and 2007 are the same within the adopted conservative photometric uncertainty $(0.1)$. Despite the photometric uncertainties, the colour indices of star W77-6a and the 2005 star W77-6b are suited for the following analysis. The position of star W77-6a adjacent to W77-8 in the $J-H / H-K$ s diagram indicates that it is a slightly reddened early A-type star. W77-6b has a NIR excess which indicates a circumstellar disk. In the optical the W77-6 binary is associated with a bright nebulous patch. In the Spitzer images W77-6 displays a complex shape where a nebulosity varying in shape and size is seen at the position of star W77-6a. Neither of the W77-6 components was detected in X-rays (GFLBG). W77-6 is further discussed in Sect. 4.3 below.

\subsubsection{Star 2MASS J13574059-4000590}

This bright star (2MASS $J, H$, and $K$ s magnitudes 9.44, 8.24, and $7.75 \mathrm{mag}$, respectively) lies near the CG $12 \mathrm{SW}$ core. It is outside the analysed SOFI image but is seen at the southern 
corner in Fig. 2. The $(J-H)$ and $(H-K s)$ colours are 1.2 and $0 \mathrm{~m} .49$, and if it were assumed to be a main sequence star its visual extinction $A_{\mathrm{v}}$ would be $4^{\mathrm{m}}$ or more. As the strong reddening starts first at the distance of CG 12 (Maheswar et al. 1996), the star is either embedded in CG 12 or located behind the cloud. If embedded its calculated dereddened absolute $K$ s magnitude would be approximately $-2^{\mathrm{m}}$. The star is either a very early type object or, more likely, a late-type giant behind the cloud. But spectroscopic observations are needed to clarify whether the star is a late-type giant or an early type star. In the Spitzer images the star is saturated in all but the $8 \mu \mathrm{m}$ band.

\subsubsection{Chandra X-ray detections}

GFLBG argue that half of the 128 Chandra X-ray detections in the direction of CG 12 are likely to be T-Tau stars associated with CG 12. The detections within the SOFI image coincide with stars 74, 86/87, 108, h4636n/h4636s, 41 (J 135732.16-395836.3, I_36), 83 (J 135738.21-395947.7, I_42) and 216 (J 1357537.4395730.8, I_68). The first six stars have been discussed above. Of the last three only star 83 has significant extinction $\left(A_{\mathrm{V}} \sim\right.$ $7^{\mathrm{m}}$ ) and the other two lie near the main sequence. The observed apparent magnitudes of these stars are in agreement with them being at the assumed distance of CG 12 (550 to $630 \mathrm{pc}$ ).

Of the remaining five GFLBG X-ray detections in the SOFI field one (I_37) coincides with a galaxy and four (I_31, I_40, I_63 and I_71) have no NIR counterpart in the SOFI images. These four are extragalactic X-ray candidates. The GFLBG X-ray detections I_23 (2MASS J13572321-3957498), I_27 (2MASS J13572583-3958372), I_29 (2MASSJ135726013956271), I_47 (2MASS J13573973-3954035), and I_61 (2MASS J13574510-4001071) coincide with stellar sources at the cropped-off edges of the SOFI images and are identified in Fig. 2. Of these five stars SOFI three-colour data are available only for I_29 and I_47. Even though the star I_47 is at the edge of the SOFI images where reliable photometry is difficult, the photometry is in agreement with the 2MASS magnitudes (maximum deviation 0.07 ). The situation is different for I_29. The SOFI and 2MASS JHKs magnitudes are $14.10,13.02,12^{\mathrm{m}} .48$ and $14.84,13.44,12 \mathrm{~m} 63$ respectively. The difference is much higher than the one observed for I_29, even though the two stars are of similar brightness. The observed Chandra source median energy of $1.2 \mathrm{keV}$, which indicates a low absorbing column density, was considered unusual by GFLBG because the 2MASS photometry $((J-H)=1 \mathrm{~m} .44,(H-K \mathrm{~s})=0.81)$ infers a visual extinction of $\sim 6^{\mathrm{m}}$, which is higher than expected as well as NIR excess. The SOFI photometry $\left((J-H)=1^{\mathrm{m}} .08,(H-K \mathrm{~s})=0.54\right)$ does not indicate NIR excess and the inferred extinction is lower. The star is probably variable.

\subsubsection{Potential cluster member stars}

As discussed above, all stars in the SOFI $K$ s image with apparent or de-reddened magnitude brighter than $\sim 15^{\mathrm{m}}$ are possible members of the cluster associated with CG 12 . However, for most of the stars $J H K$ s photometry alone is not sufficient to discriminate between cluster members and field stars. NIR excess and an associated nebulosity or X-ray activity of some of the stars confirms their membership.

The location of many of the stars which are brighter than $15^{\mathrm{m}} 0$ in $K \mathrm{~s}$ (marked with red circles) in the $J-H / H-K$ s diagram (Fig. 3) indicates extinction up to a few magnitudes in the visual, showing that they are either embedded in CG 12 or are background stars. Some of these could be T-Tauri stars which were not active during the GFLBG Chandra observations. The GFLBG objects I_36 and I_68 (stars 41 and 216) lie near the unreddened main sequence. A group of bright stars lies near $(J-H),(H-K \mathrm{~s}) 1.7,0.8$ in the $J-H / H-K$ s diagram. Apart from the X-ray sources I_41 and I_42 (stars 74 and 83) and star 120 three other stars (76, 93 and 99) belong to the group. These stars are potential cluster members, but further, e.g. spectroscopic, observations are needed to confirm the membership.

In addition to stars already discussed above, star 80 is the only star for which $J H K$ s colours indicate clear NIR excess. The star is, however, much fainter than e.g. the two other stars (87 and 108) with strong NIR excess.

\subsubsection{Visual extinction}

The NICE method presented in Lada et al. (1994) and the SOFI NIR photometry can be used to evaluate the visual extinction within the CG 12 cloud. However, the location of CG 12 high above the Galactic plane is not favourable for applying the NICE method. According to the Mattila (1980) model the expected number of stars behind CG 12 up to a distance of $1 \mathrm{kpc}$ is only 19. Like the foreground stars these are of the spectral type G5 or later. If the stars are distributed randomly in the SOFI image, not many are expected in the direction of the likely positions of the highest extinction, CG 12-S and CG 12-N. Because of the high surface brightness in these positions, the $J$ limiting magnitude is also lower than the value $21 . \mathrm{m} 0$ quoted for the SOFI image as a whole in Sect. 2. One would therefore not expect $J$ band detections of background stars lying farther than $1 \mathrm{kpc}$ in the directions of high $\left(>20^{\mathrm{m}}\right)$ extinction. After eliminating the known CG 12 cluster members and the few assumed foreground stars, the visual extinction in the cloud could be estimated. However, as argued above, the SOFI data are not deep enough to probe the visual extinction through the two densest cloud cores, CG 12-N and CG 12-S. The observed extinction reaches to $\sim 20^{\mathrm{m}}$ around $\mathrm{CG} 12-\mathrm{N}$ and $\sim 15^{\mathrm{m}}$ around $\mathrm{CG} 12-\mathrm{S}$. The lower measured extinction towards CG 12-S is most probably due to the very bright surface emission around h4636 and the cone (Fig. 1). which is the reason for the limiting magnitude to be even lower than in the direction of CG 12-N. There are no background stars bright enough to be observed through the core centres, and therefore only a lower limit for the visual extinction is obtained. As predicted, the stars suffering the highest extinction are $H, K$ s detections only. The $J-H / H-K$ s diagram in Fig. 3 indicates that the extinction does not reach zero within the SOFI image. This is to be expected as the observed optical CG 12 surface brightness extends far outside the SOFI image (see, e.g., Fig. 8 in Paper 1).

\subsection{Surface brightness}

Apart from the two nebulous bright binaries in the SOFI field the most intriguing feature in the image is the cone-shaped nebulosity SW from h4636. Indication of the bright cone opening to the North is already faintly seen in the 2MASS images, but the fainter counter cone opening to the South is only seen in the SOFI $K$ s image (see Fig. 4). Together the cones form an hourglass-shaped object with a bright point at the "waist". This object is listed as a 2MASS source 2MASS J135741473959252. This source is not stellar-like in the SOFI JHKs images but blends into the surface brightness. The cone and the central source are visible in the Spitzer 3.6 and $4.5 \mu \mathrm{m}$ images. 


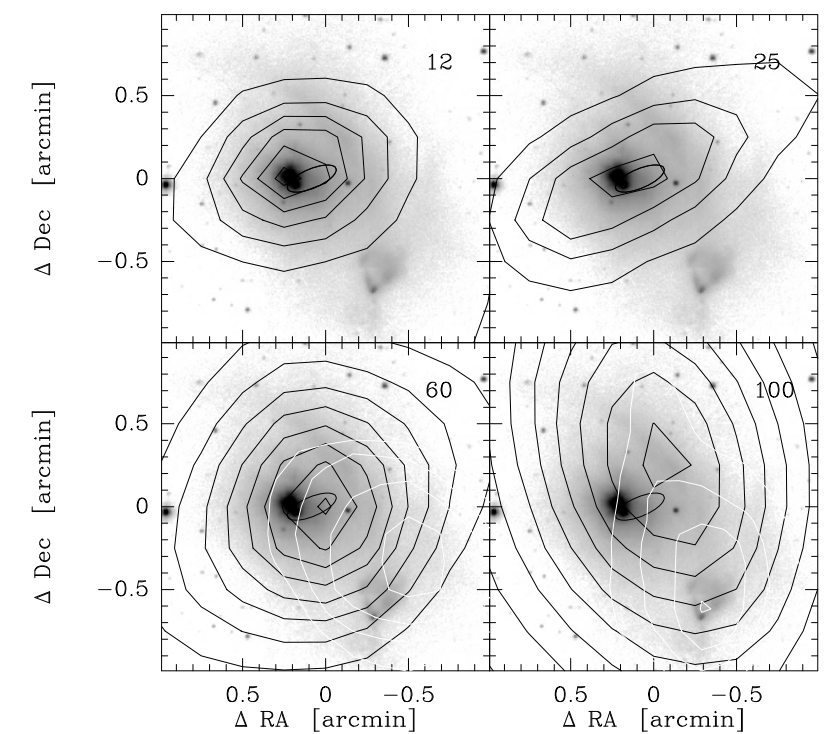

Fig. 4. Contour maps (arbitrary contour values) of the HIRES processed IRAS maps superposed on the SOFI $K$ s band image. The IRAS 13547-3944 positional uncertainty ellipse is also shown. The map wavelength is shown in microns in the upper right corner of each panel. Superposed using white contours on the $60 \mu \mathrm{m}$ panel are the $\mathrm{C}^{18} \mathrm{O}(2-1)$ integrated emission contours (Paper 1) and on the $100 \mu \mathrm{m}$ panel the $1.2 \mathrm{~mm}$ continuum emission (Haikala 2010, in preparation). The offset in arcminutes from the IRAS 13547-3944 point source position is shown on the axis.

The central source is still visible in the $6 \mu \mathrm{m}$ image but the cone blends into the background. At $8 \mu \mathrm{m}$ only background nebulosity is seen. The cone is projected on the $\mathrm{DCO}^{+}$core and the $1.2 \mathrm{~mm}$ continuum source (Paper 1; Paper 2) and it coincides with the centre of the collimated molecular outflow detected by White (1993). The hourglass is therefore most probably a cavity cleared by the outflow where the walls are reflecting light from the outflow central source. A long cavity opening north of the bright cone is best seen in the $J$ image. It corresponds to a cavity vacated by the outflow into the less tenuous part of the dust/molecular cloud. A similar but less pronounced feature is seen also south of the hourglass.

The north-south dark lane between star 74 and the binary $86 / 87$ is likely to be a shadow cast by the star 87 circumstellar disk and not due to obscuration in the dust cloud. Such shadows were first recognised by Hodapp et al. (2004) in NGC1333. The position of the shadow with respect to the probable circumstellar disk around star 87 corresponds to the theoretical Pontoppidan \& Dullemond (2005) scenario 2a in which a shadow cast by the disk is seen on the surface of an adjacent dust cloud.

The NIR surface brightness is a powerful tool for studying the structure of isolated dark cloud cores illuminated by the diffuse interstellar radiation field (ISRF). For an ideal spherical dust cloud the $J, H$, and $K$ surface brightness due to reflected ISRF forms concentric rings where the radius gets smaller the longer the wavelength. The inner radius of each ring corresponds to the optical depth of approximately 1 . The phenomenon has been observed e.g. by Lehtinen \& Mattila (1996) and discussed in detail in Juvela et al. (2008). CG 12 does not correspond to this ideal case as because apart from the ISRF the cloud also shows a strong internal source of light (h4636). The binary in CG 12-N (stars 86 and 87) and W77-6 also act as smaller and more localised sources of light. Offset from these light sources there are three localised positions where the long wavelength emission becomes strong, and the surface brightness turns redder in Fig. 1. These positions are south of the cone, east of the binary $86 / 87$ and around star 58. The first two coincide with the millimetre continuum sources (Paper 1), exactly in positions where one would expect the phenomenon.

\subsubsection{Spitzer NIR imaging}

The Spitzer imaging covers a much larger area than the SOFI observations presented in this paper. In the future the longer wavelength IRAC observations will provide additional information for stars inside the SOFI field and, will in particular allow the construction of a meaningful NIR spectral energy distribution from $1 \mu \mathrm{m}$ to $8 \mu \mathrm{m}$ for many stars. This will e.g. permit the separation of the background objects from the list of potential member stars in Table 1 as well as a separation of the Class I and II objects. Apart from the objects already discussed above, the new Spitzer imaging does not provide new obvious bright CG 12 cluster member candidates in the SOFI field.

The probable driving source of the collimated molecular outflow (White 1993) is better resolved in the Spitzer data than in the SOFI $J H K$ s images, where the object is heavily blended with the background nebulosity. A major part of the object in the waist of the "hourglass" in the SOFI $H$ and $K$ s images is not direct emission from the central object, but light reflected from its surroundings.

In the SOFI data the binary W77-6 does have a surrounding nebulosity, but the binary itself can be well resolved. The Spitzer data show a complicated nebulous structure which varies with wavelength and a bright stellar-like source north of W77-6b. The source is very bright in the $5.8 \mu \mathrm{m}$ image.

\subsection{IRAS point sources}

CG 12 was observed by the IRAS satellite at wavelengths between 12 and $100 \mu \mathrm{m}$. The spatial resolution of the original IRAS images can be enhanced using HIRES processing which uses the maximum correlation technique (Auman et al. 1990). The early HIRES processed maps had often wrong coordinate zero points. The HIRES maps presented in White (1993) are offset to the South from the correct position. The spatial resolution of the 60 and $100 \mu \mathrm{m}$ IRAS maps, even if enhanced with the HIRES processing, is not sufficient to resolve details in CG 12 (see White 1993, Fig. 3). The mid- and far-IR emission in CG 12 is dominated by the strong point source IRAS 13547-3944 near h4636. The second much fainter point source in CG 12 is IRAS 13546-3941. Its nominal position is slightly offset to the southeast from the $\mathrm{C}^{18} \mathrm{O}$ maximum CG $12-\mathrm{N}$ and a millimetre continuum source (Paper 2). The IRAS small extended source $\mathrm{X} 1354-397$ is seen in the direction of the $\mathrm{DCO}^{+}$core.

Two further IRAS point sources are associated with CG 12. IRAS 13543-3941 lies west of CG 12-N towards the star W77-8 and the optical nebulosity Bernes 146. IRAS 13549-3950 south of the SOFI image lies towards the star W77-2 and an optical reflection nebulosity.

\subsubsection{IRAS $13547-3944$}

IRAS 13547-3944 has been frequently associated with the binary h4636. However, the binary lies east of the point source outside its positional uncertainty ellipse. The observed emission line spectrum of h4636n and the infrared excess deduced from 
the JHKL photometry containing both the $n$ and $s$ components led Williams et al. (1977) to model the binary with a B7 star (h4636s) plus a B4 star (h4636n) with a warm (1600 K) circumstellar shell. The point source fluxes are 7.8, 8.9, 67.5, 201.9 Jy at $12,25,60$ and $100 \mu \mathrm{m}$, respectively. The IRAS fluxes at 12 and $25 \mu \mathrm{m}$ could be appropriate for a warm circumstellar shell. In the ISO-LWS FIR colour-colour diagram, IRAS 13547-3944 lies in the region occupied by Class 0 sources well offset from HAEBE objects (Pezzuto et al. 2002). This and the IRAS 60 and $100 \mu \mathrm{m}$ fluxes point at a cold cloud core and not to a warm circumstellar shell. The highest contours of the HIRES processed IRAS emission in the 12 to $100 \mu \mathrm{m}$ bands is shown superposed on the SOFI $K \mathrm{~s}$ band image in Fig. 4. The white contours in the $60 \mu \mathrm{m}$ and the $100 \mu \mathrm{m}$ panels trace the $\mathrm{C}^{18} \mathrm{O}(2-1)$ integrated emission (Paper 1) and the $1.2 \mathrm{~mm}$ continuum emission (Haikala 2010, in preparation) respectively. The IRAS 13547-3944 point source position uncertainty ellipse is drawn in all the panels.

Figure 4 is in accord with a confused situation where two independent sources, one warm and one cold, are separated by less than the IRAS beam at $12 \mu \mathrm{m}$. Given the IRAS low spatial resolution ( $30^{\prime \prime}$ at $12 \mu \mathrm{m}$ and about $2^{\prime}$ at $\left.100 \mu \mathrm{m}\right)$, h4636n has been in the IRAS beam and has contributed to the point source flux and to also to the point source position at least at $12 \mu \mathrm{m}$. The hypothetical cold source would be well within the IRAS FIR (and ISO-LWS) beams. The $1.2 \mathrm{~mm}$ continuum maximum, which coincides with the $\mathrm{DCO}^{+}$core and the NIR cone, lies SW of the nominal position of the point source and from the maxima of IR emission in all the IRAS bands. However, the $1.2 \mathrm{~mm}$ continuum emission is elongated towards the IRAS $100 \mu \mathrm{m}$ maximum. It is not likely that star 108 is the cold IRAS 13547-3944 source. Star 108 could contribute to the 12 and $25 \mu \mathrm{m}$ IRAF fluxes but the ISO-LWS data point at a cold source at an earlier phase of evolution.

\subsubsection{IRAS 13546-3941}

This point source, which lies near the tip of the globule and between the binaries W77-6a/b and $86 / 87$, is a $25 \mu$ m-detection only (1.38 Jy) and only upper limits are given in the three other IRAS bands. HIRES processed IRAS 12 (in red) and $25 \mu \mathrm{m}$ (in black) contour maps of CG $12-\mathrm{N}$ superposed on the SOFI $K \mathrm{~s} \mathrm{im-}$ age are shown in Fig. 5. The blue contours trace the $1.2 \mathrm{~mm}$ continuum emission maximum (Haikala 2010, in preparation). The positional uncertainty ellipse of IRAS 13546-3941 is also shown. The $12 \mu \mathrm{m}$ map has two maxima, one around the binary $86 / 87$ and another just to the side of the binary W77-6. Both maxima lie outside the IRAS point source positional uncertainty error ellipse. In the $25 \mu \mathrm{m}$ map the emission is elongated and encompasses both binaries, but the maximum lies between the binaries near the $\mathrm{C}^{18} \mathrm{O}$ maximum $\mathrm{CG} 12-\mathrm{N}$ and the $1.2 \mathrm{~mm}$ continuum source which is indicated with the blue contour in Fig. 5. There is no clear indication of 12 or $25 \mu \mathrm{m}$ emission in the direction of star 58 .

The IR excess of star 87 in the $J-H / H-K$ s diagram and the probable disk shadow west of it is a strong indication of a circumstellar disk. One would expect the disk to be visible at $12 \mu \mathrm{m}$. The $J H K$ s colours of W77-6b indicate NIR excess and a circumstellar disk or shell. A strong nebulosity surrounds W77-6 in the Spitzer 5.8 and $8 \mu \mathrm{m}$ images, possibly indicating a further source which is not seen in the NIR SOFI images. This putative source and/or the W77-6b circumstellar shell/disk could explain the $12 \mu \mathrm{m}$ HIRES maximum near the star. The HIRES $25 \mu \mathrm{m}$ maximum, which nearly coincides with the IRAS 13546-3941 point source position, lies between the binaries and coincides

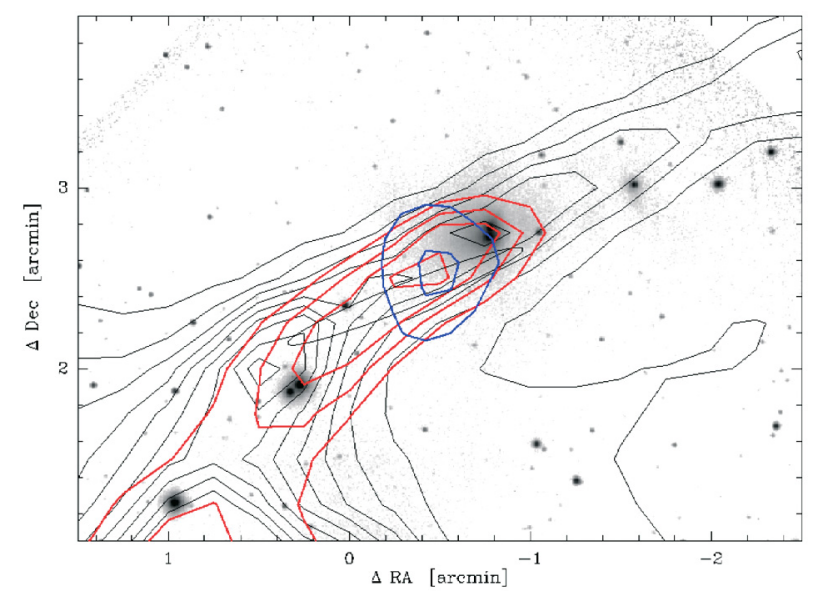

Fig. 5. Contour maps (arbitrary contour values) of the HIRES processed IRAS $12 \mu \mathrm{m}$ (black contours) and $25 \mu \mathrm{m}$ (red contours) maps of the CG 12-N region superposed on the SOFI $K$ s band image. The blue contour traces the maximum of the $1.2 \mathrm{~mm}$ continuum emission (Haikala 2010, in preparation). The IRAS $13546-3941$ positional uncertainty ellipse is indicated by the ellipse. Axis offsets are as in Fig. 4.

with the $\mathrm{C}^{18} \mathrm{O}$ maximum $\mathrm{CG} 12-\mathrm{N}$, and the $1.2 \mathrm{~mm}$ continuum core.

The location of the HIRES $12 \mu \mathrm{m}$ maxima with respect to the NIR excess stars 87 and W77-6b indicates strongly that these are real $12 \mu \mathrm{m}$ sources and not artifacts of the HIRES processing. Future mid- and far-IR observations with better spatial resolution than was possible with the IRAS satellite are, however, necessary to confirm this.

\section{Summary and conclusions}

The head of the cometary globule CG 12 has been observed in the $J, H$, and $K$ s bands. The new NIR photometry combined with already existing data at other wavelengths allows the analysis of the star formation and the content of the associated stellar cluster in more detail than was possible before.

Star formation in CG 12 (within the region imaged in $J H K$ s with SOFI) is concentrated on and near the two strong $\mathrm{C}^{18} \mathrm{O}$ maxima CG $12-\mathrm{S}$ and CG $12-\mathrm{N}$. Apart from the known members of the associated stellar cluster several new, embedded cluster members were detected as well. Because the SOFI images are much deeper than the available 2MASS survey data it is possible to define more accurately the NIR colours of the already known embedded member stars which were near the 2MASS limit.

Apart from the stellar photometry, the SOFI imaging allows the study of the cloud surface brightness due to scattered light. Scattered light permits us to pinpoint the central source of the highly collimated molecular outflow detected by White (1993) and to detect the probable shadow cast by the circumstellar disk around star 87.

Using NIR $J, H, K$ s photometry alone is not in all cases sufficient to decide whether some stars are indeed members of the CG 12 stellar cluster. In some cases an associated nebulosity can confirm the membership. For four stars the observed X-ray activity (GFLBG) indicates that the stars are likely members.

The conclusions are the following:

1. Seven embedded (proto)stars (58, 74, 86, 87, 108, 120 and W77-6b) are identified. The visual extinction of these stars ranges from 10 to more than 30 mag. The stars 86 and 87 
were already known members. Stars 74, 86, 87 and 108 are also detected in X-rays (GFLBG).

2. Stars 58, 87, 108, 120, and W77-6b have infrared excess indicating a circumstellar shell or disk. The shadow of the probable disk around star 87 is seen in the cloud surface brightness.

3. Scattered light from the probable central source of the collimated outflow detected by White (1993) as well as an hourglass-shaped cavity in the parental molecular cloud formed by the outflow are detected. Apart from the hourglass a larger scale cavity cleared by the outflow to the less tenuous part of the molecular cloud is also seen. The stellar source is better resolved in the Spitzer IRAC images at 3.4, 4.5 and $5.8 \mu \mathrm{m}$ than in the SOFI $H$ and $K$ s images, where it blends into the bright background nebulosity.

4. The Williams et al. (1977) star W77-6 is a binary with a separation of $\sim 4^{\prime \prime}$. The brighter component, W77-6a is an early A type star with a visual extinction of $\sim 3$ mag. The fainter component, W77-6b, has NIR excess indicating circumstellar material. The W77-6b $J$ magnitude changed by $0{ }^{\mathrm{m}} 6$ between two observing nights. The Spitzer NIR imaging suggests that a third source and an associated nebulosity not visible in the $J H K$ s imaging may be present.

5. HIRES enhanced IRAS 12 and $25 \mu \mathrm{m}$ maps combined with IRAS fluxes and the NIR imaging suggest that the IRAS point source 13547-3944 consists of two separate sources. The 12 and $25 \mu \mathrm{m}$ emission originates in the $\mathrm{h} 4636$ $\mathrm{n}$ component and the longer wavelengths from an adjacent, embedded cold cloud source. The $12 \mu \mathrm{m}$ HIRES map of IRAS 13546-3941 resolves into two previously undetected sources coinciding with the binary stars W77-6a/b and 86/87. The HIRES $25 \mu \mathrm{m}$ maximum lies between the $12 \mu \mathrm{m}$ sources near the IRAS 13546-3941 nominal position and the $\mathrm{C}^{18} \mathrm{O}$ maximum and a mm continuum source. The $25 \mu \mathrm{m}$ detection could be a source embedded in the continuum core.

6. The SOFI data are not deep enough to probe the visual extinction through the two dense cloud cores in the direction of CG $12-\mathrm{N}$ and CG $12-\mathrm{S}$. The observed maximum extinction around CG $12-\mathrm{N}$ is $\sim 20^{\mathrm{m}}$, and $\sim 15^{\mathrm{m}}$ around CG $12-\mathrm{S}$. No background stars are seen through the core centres.
Acknowledgements. Joao Yun is thanked for providing the ISAAC K and L images of CG 12-S. It is a pleasure to thank the NTT team for the support during the two observing runs. B.R. acknowledges support by the National Aeronautics and Space Administration through the NASA Astrobiology Institute under Cooperative Agreement No. NNA04CC08A issued through the Office of Space Science. This research has made use of the SIMBAD database, operated at CDS, Strasbourg, France, and of NASA's Astrophysics Data System Bibliographic Services.

\section{References}

Ascenso, J., Alves, J., Vicente, S., \& Lago, M. 2007, A\&A, 199

Auman, H., Fowler, J., \& Melnyk, M. 1990, AJ, 99, 1674

Bertin, E., \& Arnouts, S. 1996, A\&AS, 117, 393

Bessell, M., \& Brett, J. 1988, PASP, 100, 1134

Bourke, T., Hyland, A., Robinson, G., James, S., \& Wright, C. 1995, MNRAS, 276, 1067

Chelli, A., Cruz-Gonzalez, I., \& Reipurth, B. 1995, A\&A, 114, 135

Gahm, G., \& Malmort, A. 1980, A\&A, 82, 295

Getman, K., Feigelson, E., Lawson, W., Broos, P., \& Garmire, G. 2008, ApJ, 673,331

Haikala, L., \& Olberg, M. 2007, A\&A, 466, 191

Haikala, L., Juvela, M., Harju, J., et al. 2006, A\&A, 454, L71

Hawarden, T., \& Brand, P. 1976, MNRAS, 175, 19P

Hodapp, K., Walker, C., Reipurth, B., et al. 2004, ApJ, 601, L79

Juvela, M., Pelkonen, V.-M., Padoan, P., \& Mattila, K. 2008, A\&A, 480, 445

Lada, C., Lada, E., Clemens, D., \& Bally, J. 1994, ApJ, 429, 694

Lehtinen, K., \& Mattila, K. 1996, A\&A, 309, L570

Maheswar, G., Manoj, P., \& Bhatt, H. 1996, MNRAS, 355, 1272

Marraco, H., \& Forte, J. 1978, ApJ, 224, 473

Mattila, K. 1980, A\&AS, 39, 53

Meyer, M., Calvet, N., \& Hillenbrand, L. 1997, AJ, 114, 288

Persson, S. E., Murphy, D. C., Krzeminski, W., Roth, M., \& Rieke, M. J. 1988, AJ, 116, 2475

Pezzuto, S., Grillo, F., Benedettini, M., et al. 2002, A\&A, 330, 1034

Pontoppidan, K., \& Dullemond, C. 2005, A\&A, 435, 595

Reipurth, B. 1983, A\&A, 117, 183

Reipurth, B., \& Zinnecker, H. 1993, A\&A, 278, 81

Reipurth, B., Bally, J., \& Walawender, J. 2008, Handbook of Star Forming Regions, Volume II: The Southern Sky, ASP Monograph Publications, ed. B. Reipurth, ASP, 847

van Till, H., Loren, R., \& Davis. 1975, ApJ, 198, 235

White, G. 1993, A\&A, 274, L33

Williams, P., Brand, P., Longmore, A., \& Hawarden, T. 1977, MNRAS, 181, 179

Yonekura, Y., Hayakawa, T., Mizuno, N., et al. 1999, PASJ, 51, 837 